\title{
Materiales híbridos moleculares orgánicos-inorgánicos: síntesis y aplicación como electrodos en baterías recargables de litio
}

\author{
G. TORRES-GÓMEZ, M. LIRA-CANTÚ Y P. GÓMEZ-ROMERO \\ Institut de Ciència de Materials de Barcelona (CSIC). Campus UAB, 08193 Bellaterra, Barcelona.
}

\begin{abstract}
Se describe la síntesis y aplicación como electrodos para el almacenamiento o conversión de energía de materiales híbridos basados en la dispersión de especies inorgánicas electroactivas en el seno de polímeros orgánicos conductores. Polianilina y polipirrol son dopados con polioxometalatos electroactivos $\left(\left[\mathrm{PMo}_{12} \mathrm{O}_{40}\right]^{3-}\right)$ o aniones ferricianuro $\left(\left[(\mathrm{FeCN})_{6}\right]^{3-}\right)$ como únicas especies dopantes. La elevada carga y tamaño de estos aniones evitan, en la mayoría de los casos, su desintercalación durante la reducción. Estos híbridos se han sintetizado por métodos químicos y electroquímicos, siendo la relación anillos de anilina o de pirrol por anión de $\left[\mathrm{PMo}_{12} \mathrm{O}_{40}\right]^{3-}$ de nueve, de manera que el anión permanece en el interior de la matriz polimérica incluso después de reducir el material a $-0.4 \mathrm{~V}$ (vs Ag/ $\mathrm{AgCl}, 2.6 \mathrm{~V}$ vs Li). En el caso del híbrido Polipirrol / Fe(CN) 6 la relación anillos de pirrol/anión oscila entre 10 y 12 dependiendo de la temperatura de síntesis. La temperatura también afecta a su conductividad eléctrica, oscilando los valores más elevados alrededor de $60 \mathrm{Scm}^{-1}$ (preparado a $0^{\circ} \mathrm{C}$ ). El anión permanece en el interior de la matriz polimérica cuando se reduce en medio orgánico. Los híbridos con PMo12 pueden intercalar hasta 5.3 $\mathrm{Li}^{+}$durante la descarga (52Ah/Kg). El híbrido con $\mathrm{Fe}(\mathrm{CN})_{6}$ intercala 2.7 iones litio por fórmula unidad $(69 \mathrm{Ah} / \mathrm{Kg})$.
\end{abstract}

Palabras clave: Hibridos orgánico-inorgánicos, polimeros conductores, polioxometalatos, hexacianoferrrato, baterías recargables de litio

Molecular Organic-Inorganic Hybrid Materials : Synthesis and Application as Electrodes in Lithium Rechargeable Batteries

A novel family of molecular hybrid materials based on electroactive inorganic species dispersed in conducting organic polymers is reported as electrodes for energy storage or conversion. Polyaniline and polypyrrole are effectively doped with electroactive polyoxometalates $\left(\left[\mathrm{PMo}_{12} \mathrm{O}_{40}\right]^{3-}\right)$ or ferricyanide $\left(\left[(\mathrm{FeCN})_{6}\right]^{3-}\right)$ anions as the only doping species. The high charge and size of these anions prevents their deintercalation during reduction in most cases. The synthesis of these hybrids can be made by chemical (bulk powders) and electrochemical (films) methods. For $\left[\mathrm{PMo}_{12} \mathrm{O}_{40}\right]^{3-}$, nine aniline/pyrrole rings by anion are found in each case and the anion stays in the polymer matrix even after reduction at $-0.4 \mathrm{~V}$ ( $\mathrm{vs} \mathrm{Ag} / \mathrm{AgCl}, 2.6 \mathrm{~V} \mathrm{vs}$ $\mathrm{Li})$. In the case of Polypyrrole-FeCN 6 hybrid the pyrrole-ring $/ \mathrm{Fe}(\mathrm{CN})_{6}$ ratio was around $10-12$ depending on the temperature of synthesis. Temperature also affects the electrical conductivity, with the best values around $605 \mathrm{~cm}^{-1}$ (sample prepared at $\left.0^{\circ} \mathrm{C}\right) . \mathrm{Fe}(\mathrm{CN})_{6}$ stays in the polymer matrix when the hybrid material is reduced in organic media. $\mathrm{PMo}_{12}$ hybrids intercalate up to $5.3 \mathrm{~h}^{+}$during discharge $\left(52 \mathrm{Ah} / \mathrm{Kg}\right.$ ) whereas the hybrid with $\mathrm{Fe}(\mathrm{CN})_{6}$ intercalates 2.7 lithium ions per formula unit (69Ah/Kg).

Keywords: Organic-inorganic hybrids, conducting polymers, polyoxometalates, hexacyanoferrate, rechargeable lithium batteries

\section{INTRODUCCIÓN}

La combinación única de propiedades físicas que se da en los polímeros orgánicos conductores (POC) los ha convertido en objeto de un gran número de estudios en los últimos años. Los POC se han estudiado básicamente como conductores moleculares, pero también como componentes en todo tipo de dispositivos electroquímicos y fotoelectroquímicos, en baterías, supercondensadores, sensores, fotoconductores o celdas de fotoconversión (1-5).

Pero además de sus características cadenas poliméricas conjugadas, estos materiales contienen a menudo especies químicas adicionales. Así, se incorporan cationes en la estructura de sus derivados $n$-dopados y aniones en los derivados p-dopados, más frecuentes. En principio estos iones dopantes sólo realizan una labor de compensación de carga de manera que en la gran mayoría de los casos se han usado aniones simples inactivos para el dopaje de los POC.

Por otro lado, el proceso de dopaje proporciona una oca- sión única para incorporar en el seno de los POC una gran variedad de interesantes especies ionicas electroactivas o fotoactivas. En los últimos años hemos explorado la síntesis y actividad electroquímica de materiales híbridos orgánicoinorgánicos basados en POC y aniones inorgánicos electroactivos por incorporación de éstos últimos en la matriz polimérica como contraiones dopantes (6-12). Los aniones polioxometalatos del tipo $\left[\mathrm{XMo}_{12} \mathrm{O}_{40}\right]^{3-}(\mathrm{X}=\mathrm{Mo}, \mathrm{W})$ son conocidas especies activas electroquímica y fotoquímicamente. Su naturaleza molecular y su consecuente solubilidad han sido el mayor obstáculo para su aplicación en dispositivos como electrodos sólidos.

El anión fosfomolibdato es un clúster que contiene 12 átomos de $\mathrm{Mo}(\mathrm{VI})$ con actividad fotoquímica y una química redox reversible, pero con una capacidad limitada para aceptar electrones reversiblemente. Así, no puede llegar a un estado de reducción correspondiente a un electrón por átomo 
metálico de forma reversible. En cambio, otros aniones inorgánicos como el $\mathrm{Fe}(\mathrm{CN})_{6}$ sí que pueden. El par ferro/ferricianuro es un sistema modelo, capaz de intercambiar reversiblemente un electrón por átomo de hierro. Además tiene un bajo peso molecular y por lo tanto un peso electroquímico equivalente muy favorable, lo cual lo hace muy atractivo en dispositivos de almacenamiento de energía como baterías de litio o supercondensadores.

Describiremos aquí la síntesis y las propiedades electroquímicas de un nuevo tipo de materiales híbridos orgánicoinorgánicos formados por una matriz de polímero orgánico conductor (POC) y especies inorgánicas electroactivas dispersas en ella a nivel molecular. Incluimos ejemplos de híbridos basados en polioxometalatos y en hexacianoferrato. A continuación veremos cómo las propiedades electroquímicas de estas moléculas polinucleares pueden funcionar como parte de un electrodo de inserción sólido en un tipo de comportamiento cooperativo que normalmente está fuera del alcance de las especies moleculares.

\section{PROCEDIMIENTO EXPERIMENTAL}

\subsection{Reactivos}

El pirrol y la anilina (Aldrich) se destilaron al vacío y se mantuvieron en $\mathrm{N}_{2}$ a $4^{\circ} \mathrm{C}$ hasta su uso. El resto de reactivos se usaron como se recibieron. El contenido en agua de $\mathrm{H}_{3} \mathrm{PMo}_{12} \mathrm{O}_{40} \cdot 17 \mathrm{H}_{2} \mathrm{O}$ se determinó por análisis termogravimétrico.

\subsection{Medidas}

Los análisis elementales se realizaron en un aparato Carlo Erba CHN EA 1108. La resistividad eléctrica de las muestras se determinó por el método de Van der Pauw. Los estudios electroquímicos se llevaron a cabo en un potenciostato/galvanostato EG\&G PAR207A controlado por ordenador. Se usó una celda de tres electrodos con un electrodo de referencia de $\mathrm{Ag} / \mathrm{AgCl}$ y un contraelectrodo de Pt para la caracterización en medio acuoso, y Li metálico como referencia en medio orgánico.

\subsection{Análisis de baterías}

Las celdas electroquímicas reversibles (2 electrodos) se montaron en soportes Swagelok, usando una caja seca Labconco 50700 que permite trabajar en una atmósfera con niveles de agua y oxígeno inferiores a 1 y 5 ppm respectivamente. Como electrodo negativo se usó lámina de Li metálico de 13-16 mm de diámetro y $0.1 \mathrm{~mm}$ de grosor, como electrodo positivo se usó una mezcla íntima del material híbrido y carbón Super P (20\%) y como electrolito se usó una disolución de $\mathrm{LiPF}_{6} 1 \mathrm{M}$ en una mezcla 1:1 de EC:DMC o EC:DEC. Las celdas se estudiaron aplicando ciclos de carga y descarga a corriente constante en un potenciostato ARBIN modelo BT2042.

\subsection{Síntesis}

\subsubsection{SÍNTESIS DE PAni / $\mathrm{PMo}_{12}$}

$1 \mathrm{~mL}$ de anilina se añadió directamente a $5.00 \mathrm{~g}$ de $\mathrm{H}_{3} \mathrm{PMo}_{12} \mathrm{O}_{40}$ sólido. La mezcla se agitó lentamente usando una varilla de vidrio hasta que la pasta negra (y disolución azul) formadas inicialmente solidificaron para formar un polvo negro-verdoso (A). El análisis elemental da una composición: $15.27 \%$ C, $3.199 \% \mathrm{~N}, 1.545 \% \mathrm{H}$. A continuación se transfirieron $5.00 \mathrm{~g}$ del polvo (A) a un matraz con agitación magnética y flujo continuo de $\mathrm{O}_{2}$. Se controlaron el volumen de agua usado y el tiempo de reacción y se obtuvo el compuesto híbrido después de un período de al menos $24 \mathrm{~h}$ de agitación y flujo de $\mathrm{O}_{2}$ y un volumen de $40 \mathrm{~mL}$ de agua. Finalmente se obtuvo un polvo negro que se filtró y secó bajo vacío dinámico.

\subsubsection{SÍNTESIS ELECTROQUÍMICA DEL HÍBRIDO PAni/ $\mathrm{PMo}_{12}$}

Se realizó por voltamperometría cíclica usando una lámina de Pt como electrodo de trabajo, un electrodo de referencia de $\mathrm{Ag} / \mathrm{AgCl}$ y otra lámina de Pt como contraelectrodo. El polvo negro-verdoso (A) descrito en la sección de síntesis química previa se usó como reactivo inicial. De esta forma se disolvieron $5 \mathrm{~g}$ del polvo (A) en $100 \mathrm{~mL}$ de agua y se puso la disolución en una celda de tres electrodos. El material híbrido se obtuvo como una fina película sobre el electrodo de trabajo después de 12 ciclos de voltamperometría cíclica entre 0.9 y $-0.2 \mathrm{~V}$ (vs $\mathrm{Ag} / \mathrm{AgCl}$ ) a una velocidad de barrido de $1 \mathrm{mV} / \mathrm{s}$. La composición obtenida por análisis elemental para el sólido así obtenido fue: $26.390 \%$ C, $4.932 \%$ N, $1.690 \%$ H. No se pudo llevar a cabo el análisis de Mo debido a la pequeña cantidad de muestra obtenida electroquímicamente.

\subsubsection{SÍNTESIS DE PPi-Fe(CN)}

Se preparó por polimerización oxidativa del pirrol por métodos químicos y electroquímicos.

Para la síntesis química se utilizaron diversos métodos de preparación. En el mejor de ellos se añadieron $25 \mathrm{~mL}$ de una disolución 1.8M de $\mathrm{HClO}_{4}$ sobre una disolución de $3.790 \mathrm{~g}$ de $\mathrm{K}_{3}\left[\mathrm{Fe}(\mathrm{CN})_{6}\right]$ en $20 \mathrm{~mL}$ de agua. Se formó un precipitado blanco de $\mathrm{KClO}_{4}$ que se filtró, de manera que la disolución amarilla así obtenida se añadió gota a gota (durante un período de 5 minutos) sobre una disolución $0.2 \mathrm{M}$ de pirrol en agua mantenida en un baño termostatado a la temperatura deseada. En pocos segundos se empezó a formar un precipitado negro, Después de 3 minutos éste se filtró, se lavó repetidamente con agua y después con etanol y finalmente se secó al vacío durante al menos 12 horas. En otra serie de experimentos se continuó la agitación a temperatura constante durante 30 minutos antes de filtrar el sólido.

Para la síntesis electroquímica se usó una celda de tres electrodos. Los mejores resultados se obtuvieron por cronoamperometría a $0.7-0.8 \mathrm{~V}$ vs Ag/ AgCl. El electrodo de trabajo (hilo de $\mathrm{Pt}$ ) debe ser activado antes de la síntesis por aplicación de un potencial oxidante de $3 \mathrm{~V}$ durante 5 minutos en una disolución de $\mathrm{H}_{2} \mathrm{SO}_{4}$ (ac.) al 10\%. El electrolito usado para la síntesis electroquímica fue una disolución $0.1 \mathrm{M}$ de $\mathrm{K}_{4}\left[\mathrm{Fe}(\mathrm{CN})_{6}\right]$ y $0.1 \mathrm{M}$ de pirrol en agua., que no se debe agitar durante el crecimiento del film (13).

\section{RESULTADOS Y DISCUSIÓN}

La preparación de PAni o PPi tiene lugar por polimerización oxidativa del monómero correspondiente. Así, numerosos trabajos sobre PAni y sus derivados publicados en la bibliografía utilizan un medio ácido y persulfato como el agente oxidante para conseguir la polimerización. Bajo estas 
condiciones los polímeros incorporan los aniones presentes en la disolución como contraiones dopantes. Nosotros hemos aprovechado el carácter fuertemente ácido y oxidante del ácido fosfomolíbdico así como la forma ácida del hexacianoferrato para inducir directamente la polimerización de pirrol y polianilina evitando la presencia de otros aniones y, por lo tanto, obteniendo materiales con PMo12 o $\mathrm{Fe}(\mathrm{CN})_{6}$ como clústeres electroactivos anclados en el seno del polímero. Los materiales se obtuvieron tanto como polvos por métodos químicos (denominados Q) como en forma de películas o recubrimientos por métodos electroquímicos (EQ).

\subsection{Híbridos PAni-PMo12 y PPi-PMo12}

Los análisis químicos indican las siguientes composiciones para estos materiales:

PAni/ PMo12. $\left(\mathrm{C}_{6} \mathrm{H}_{4.8} \mathrm{~N}\right)_{9}\left(\mathrm{PMo}_{12} \mathrm{O}_{40}\right)(\mathrm{Q}) .\left(\mathrm{C}_{6} \mathrm{H}_{4.8} \mathrm{~N}\right)_{9.5}\left(\mathrm{PMo}_{12} \mathrm{O}_{40}\right)$. (EQ). Se obtiene una relación de nueve anillos de anilina por cada grupo PMo12 en lugar de la relación ideal 6:1

PPi/PMo12. $\left(\mathrm{C}_{4} \mathrm{H}_{3} \mathrm{NO}\right)_{9} \mathrm{PMo}_{12} \mathrm{O}_{40} \mathrm{H}_{2} \mathrm{O}(\mathrm{Q})$. $\left(\mathrm{C}_{4} \mathrm{H}_{3} \mathrm{~N}\right)_{11} \mathrm{PMo}_{12} \mathrm{O}_{40}$ (EQ). En este caso los resultados son comparables a la relación ideal máxima de 9 anillos de pirrol por PMo12

Estos materiales híbridos mantienen la naturaleza plástica de sus matrices poliméricas y presentan elevadas conductividades. Los valores óptimos son $0.5 \mathrm{Scm}^{-1}$ para materiales preparados por métodos químicos (PAni/PMo12 Q) y 1.0 $\mathrm{Scm}^{-1}$ para materiales preparados electroquímicamente (PAni/ PMo12 EQ). La conductividad para las muestras PPi/ PMo12 oscila entre 1.0 y $0.1 \mathrm{Scm}^{-1}$.

Los voltamperogramas cíclicos de estos híbridos moleculares están dominados por las ondas características del PMo12. Su actividad es más pronunciada en disoluciones acuosas ácidas que en electrolitos orgánicos. En estos últimos, las ondas redox están peor definidas e indican una reversibilidad menor.

Por ejemplo, para PAni/PMo12 en electrolito EC:DME (1:1) $/ \mathrm{LiClO}_{4} 1 \mathrm{M}$ las ondas redox correspondientes al PMo12 se pueden ver a potenciales de $2.85 / 3.2,2.68 / 2.86$ y $2.32 / 2.42$ $\mathrm{V}$ vs Li $(-0.12 / 0.23,-0.3 /-0.11 \mathrm{y}-0.65 /-0.55 \mathrm{~V}$ vs $\mathrm{Ag} / \mathrm{AgCl})$ (Ver Figura 1(a)). Los valores de intensidad máxima obtenidos para el material en electrolito orgánico muestran desplazamientos sistemáticos hacia potenciales más negativos respecto a los valores obtenidos en medio acuoso ácido (Figura $1(b))$.

De la misma forma que para $\mathrm{PPi} / \mathrm{PMo} 12$, las ondas en medio orgánico para PAni / PMo12 están desplazadas hacia potenciales inferiores respecto al medio acuoso. Un desplazamiento que crece proporcionalmente con el número de electrones añadidos en ondas sucesivas. Nosotros atribuimos ese comportamiento a la falta de protonación y consecuente incremento de carga del PMo12 en medio orgánico, cosa que hace la reducción progresivamente más difícil.

Mecanismo de inserción durante las reacciones redox: El anión fosfomolibdato es de hecho una parte integral del híbrido, tal y como se determina por análisis químicos y espectroscópicos y por su actividad electroquímica. Más aún, hemos comprobado que permanece en el seno del polímero después de una reducción. Contrariamente a lo que ocurre con otros aniones más sencillos, el anión PMo12 permanece anclado en el interior de la matriz polimérica y fuerza la inserción de cationes $\left(\mathrm{Li}^{+}\right)$dentro del híbrido.

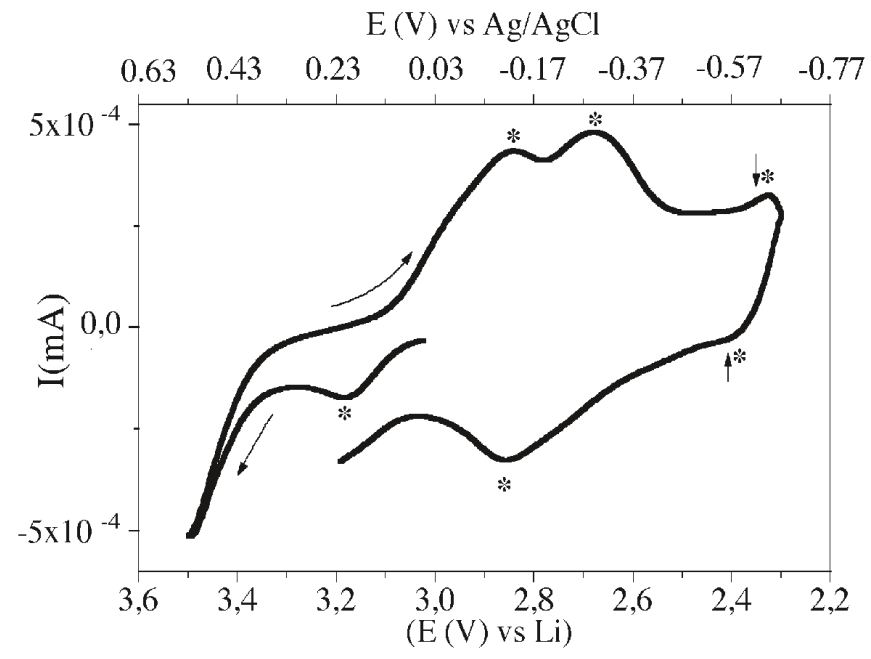

a

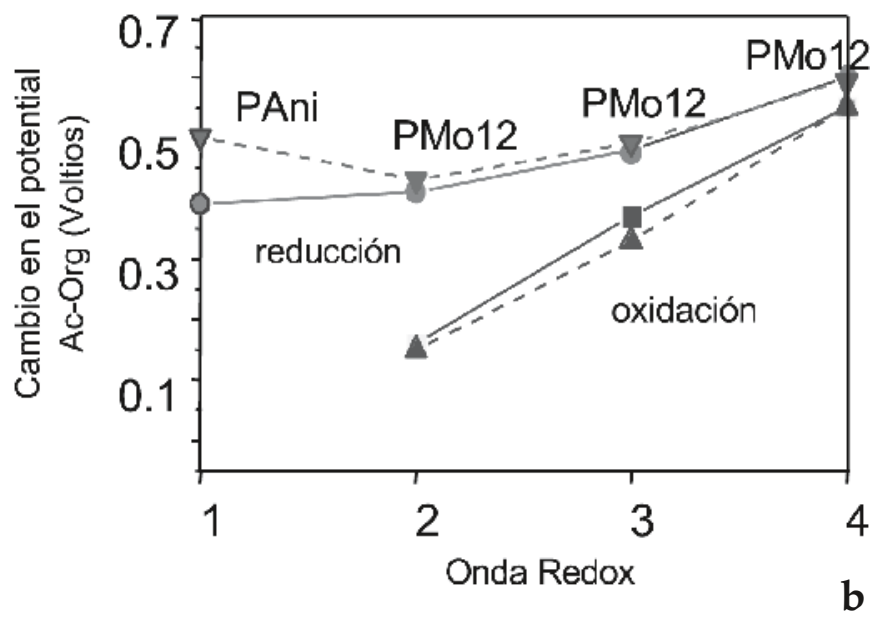

Figura 1. (a) Voltamperograma cíclico del híbrido PAni/PMo12 en electrolito EC:DME (1:1)/ $\mathrm{LiClO}_{4} 1 \mathrm{M}$. Se midió en una celda compacta de tres electrodos con Li como ánodo y electrodo de referencia (velocidad de barrido, $0.05 \mathrm{mV} / \mathrm{s}$ ). Las ondas redox señaladas con asteriscos corresponden al PMo12 mientras que las correspondientes a la PAni se señalan con flechas. (b) Diferencias entre los potenciales redox de PAni/PMo12 en medio acuoso y orgánico al aumentar el número de electrones $\quad$ = AcQOx - OrgQOx, $\quad=$ AcQRed OrgQRed, $\boldsymbol{\Delta}=$ AcEQOx - OrgQOx, $\boldsymbol{\nabla}$ = AcEQRed -OrgQRed.

Una celda reversible con PAni/PMo12 como el material activo del cátodo y Li metálico como ánodo (electrolito EC/ DME $\mathrm{LiClO}_{4} 1 \mathrm{M}$ ) generó una carga específica de $53.2 \mathrm{Ah} / \mathrm{Kg}$. Este valor corresponde a un intercambio total de 5.25 e- por fórmula unidad $\left(\left(\mathrm{C}_{6} \mathrm{H}_{5} \mathrm{~N}\right)_{9} \mathrm{PMo}_{12} \mathrm{O}_{40}{ }^{3-}\right)$. Esto está de acuerdo con el valor de átomos de Li obtenidos por análisis químico del material reducido (5.35 Li/fórmula unidad). Ambos resultados confirman el mecanismo de inserción catiónica que tiene lugar en estos electrodos híbridos moleculares.

\subsection{Híbridos $\mathrm{PPi} / \mathrm{Fe}(\mathrm{CN})_{6}$}

Se ha estudiado sistemáticamente el efecto de la temperatura y del envejecimiento sobre la conductividad de los híbri- 
dos $\mathrm{PPi} / \mathrm{Fe}(\mathrm{CN})_{6}$. En primer lugar se ha observado que se obtienen mayores conductividades en aquellos materiales preparados a temperaturas más bajas (Figura 2a). Esta relación de mayores conductividades a menores temperaturas también se ha observado en otros POC y en general se atribuye a un incremento en el número de defectos de la estructura polimérica cuando ésta se obtiene a alta temperatura, ya que estos defectos rompen la deslocalización a lo largo del sistema p. En nuestro caso hemos podido constatar además la correlación entre esta tendencia en la conductividad y un contenido más elevado del anión dopante $\mathrm{Fe}(\mathrm{CN})_{6}$ en estos materiales obtenidos a menor temperatura (Figura 2b).

Los análisis termogravimétricos de los materiales también muestran diferencias químicas significativas entre los híbridos obtenidos a distintas temperaturas. En primer lugar, hemos observado desviaciones sistemáticas de los resultados de análisis elemental. Todas las muestras presentaron valores bajos de $\mathrm{C}, \mathrm{N}, \mathrm{H}$ y Fe respecto a la fórmula ideal $[\mathrm{PPi}]_{\mathrm{x}}\left[\mathrm{Fe}(\mathrm{CN})_{6}\right]_{\mathrm{y}}$. Este hecho sería consistente con la presencia de un elemento o ion adicional en los polímeros. Otros autores (14-16) han asignado resultados similares obtenidos para diferentes derivados de PPi a la adición de grupos carbonilo e hidroxilo a la estructura del polímero cuando éste se obtiene en presencia de agua. Los análisis termogravimétricos realizados hasta $250^{\circ} \mathrm{C}$ en argón (velocidad de calentamiento, $1^{\circ} \mathrm{C} / \mathrm{min}$ ) muestran que existen dos pérdidas de peso: la primera, antes de $100^{\circ} \mathrm{C}$, se atribuye a la eliminación de moléculas de agua débilmente absorbidas en el material, mientras que la segunda, a $250^{\circ} \mathrm{C}$ es debida a la degradación del polímero. De acuerdo con estos datos y los análisis químicos, la composición de los materiales obtenidos a distintas temperaturas es:

$$
\begin{gathered}
\left(\mathrm{C}_{4} \mathrm{H}_{3} \mathrm{~N}\right)_{9.9}\left[\mathrm{Fe}(\mathrm{CN})_{6}\right] \cdot 5.2 \mathrm{H}_{2} \mathrm{O} \cdot \mathrm{O}_{5.8}\left(\mathrm{Q}, 0^{\circ} \mathrm{C}\right) \\
\left(\mathrm{C}_{4} \mathrm{H}_{3} \mathrm{~N}\right)_{10.7}\left[\mathrm{Fe}(\mathrm{CN})_{6}\right] \cdot 4.9 \mathrm{H}_{2} \mathrm{O} \cdot \mathrm{O}_{6.1}\left(\mathrm{Q}, 27^{\circ} \mathrm{C}\right) \\
\left(\mathrm{C}_{4} \mathrm{H}_{3} \mathrm{~N}\right)_{12.2}\left[\mathrm{Fe}(\mathrm{CN})_{6}\right] \cdot 6.4 \mathrm{H}_{2} \mathrm{O} \cdot \mathrm{O}_{9.6}\left(\mathrm{Q}, 49^{\circ} \mathrm{C}\right)
\end{gathered}
$$

Mecanismo de inserción durante las reacciones redox. En buen acuerdo con estudios anteriores (13,17-19), hemos observado que cuando se llevan a cabo voltamperogramas cíclicos o cronoamperometrías en medio acuoso sobre los materiales $\mathrm{PPi} / \mathrm{Fe}(\mathrm{CN})_{6}$ preparados química o electroquímicamente, existe al menos una desintercalación parcial del anión electroactivo al exterior de la matriz polimérica y hacia el electrolito acuoso, donde puede ser identificado por varios métodos.

Por otro lado, cuando los experimentos electroquímicos se llevan a cabo en un medio orgánico típicamente usado como electrolito en baterías de litio $\left(\mathrm{LiClO}_{4} 1 \mathrm{M}\right.$ en dimetoxietano:carbonato de etileno 1:1) nunca hemos detectado la presencia del anión $\mathrm{Fe}(\mathrm{CN})_{6}$ en el electrolito. Esto representa un éxito parcial en el anclaje del anión $\mathrm{Fe}(\mathrm{CN})_{6}$ en la matriz polimérica en ciertas condiciones.

Los mejores resultados obtenidos al analizar estos materiales como cátodos en celdas reversibles de litio se obtuvieron para el híbrido sintetizado a $0^{\circ} \mathrm{C}$. En este caso, la máxima carga específica obtenida fue de $69 \mathrm{Ah} / \mathrm{Kg}$, en el ciclo número 94 (Figura 3).
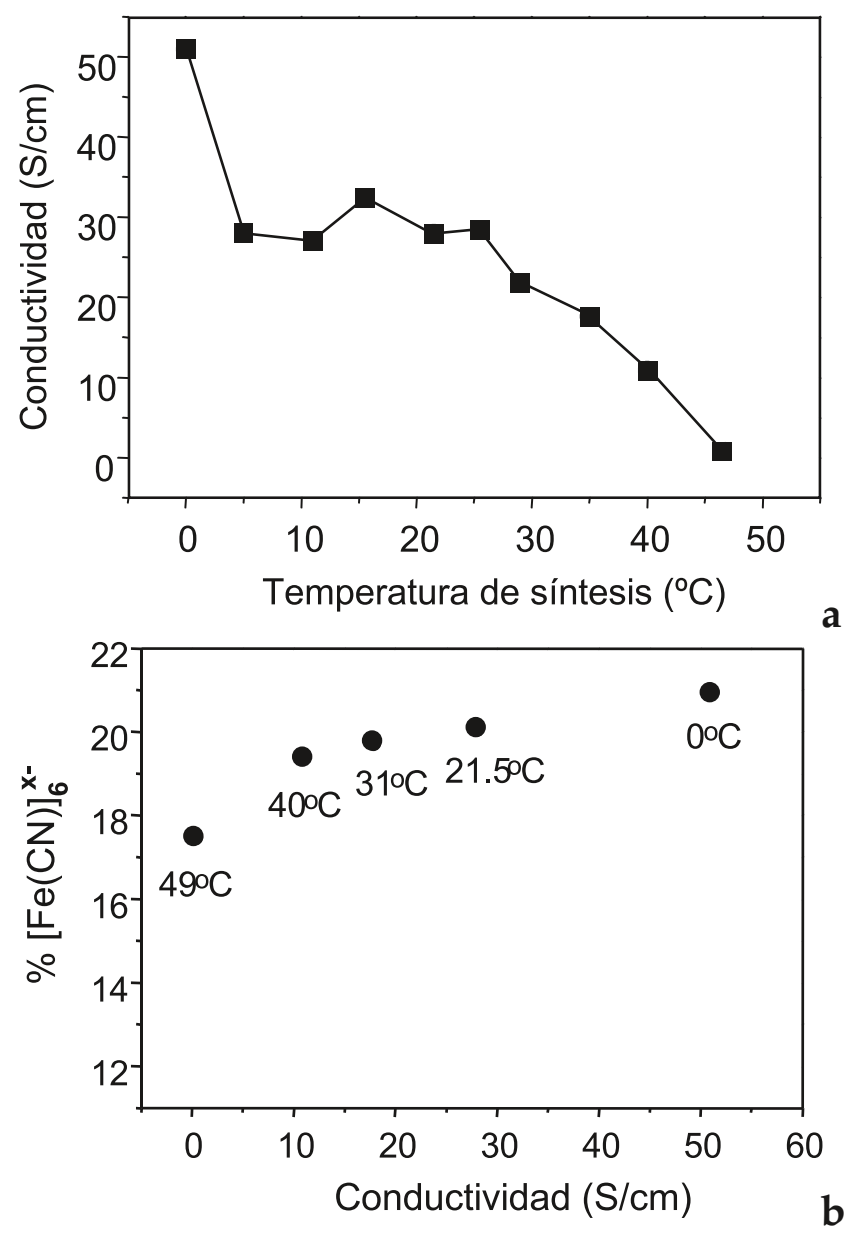

Figura 2. a) Efecto de la temperatura de reacción sobre la conductividad del híbrido PPi-Fe(CN) ${ }_{6}$. Las líneas son simplemente una guía visual. b) Relación entre conductividad y la cantidad de $\mathrm{Fe}(\mathrm{CN})_{6}$ (\% peso de $\mathrm{Fe}(\mathrm{CN})_{6} /$ peso de híbrido) para muestras obtenidas a distintas temperaturas (indicadas en la figura).

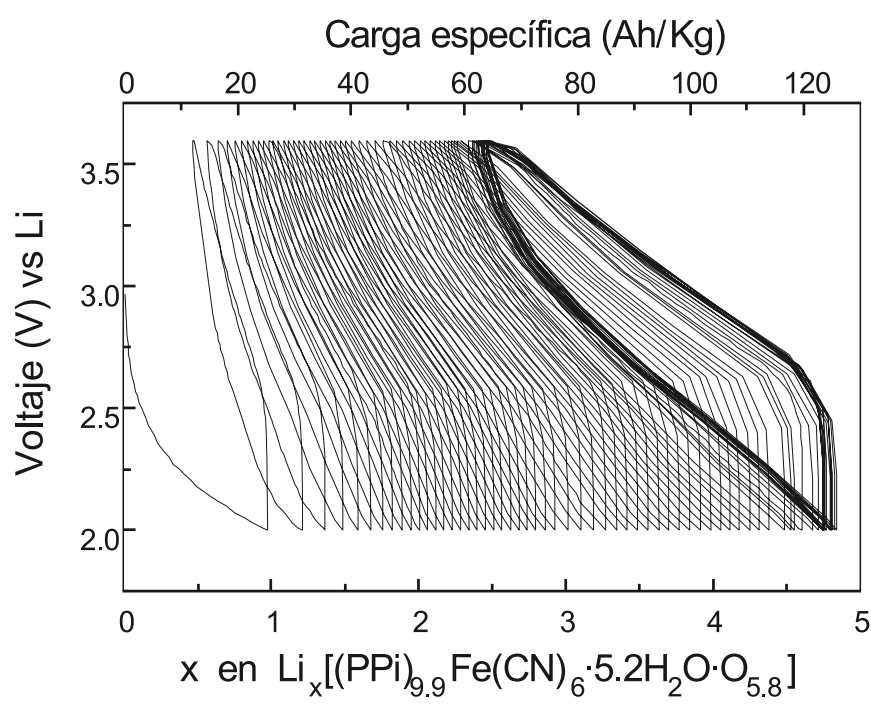

Figura 3. Primeros 51 ciclos de carga y descarga de una celda reversible de litio que usa el híbrido PPi-Fe $(\mathrm{CN})_{6}$ (obtenido a $0^{\circ} \mathrm{C}$, en forma de film con carbon Super P y PVDF) como cátodo, Li como ánodo y una disolución $\mathrm{LiPF}_{6} 1 \mathrm{M}$ en EC:DEC 1:1 como electrolito. La corriente constante aplicada fue de $25 \mu \mathrm{A}$. 


\section{CONCLUSIONES}

Hemos presentado algunas de las diversas manera de sintetizar materiales híbridos orgánico-inorgánicos a partir de polímeros orgánicos conductores y especies moleculares electroactivas. Los híbridos resultantes mantienen la buena conductividad y naturaleza plástica de la matriz polimérica a la vez que añaden la actividad específica de los componentes inorgánicos.

Estos materiales brindan una oportunidad única de aprovechar las propiedades moleculares de los aniones dopantes incorporándolos a un material sólido. Las especies moleculares aniónicas como los $\mathrm{POM}$ o el $\mathrm{Fe}(\mathrm{CN})_{6}$ se pueden anclar efectivamente en el interior del polímero aprovechando su incorporación como aniones dopantes. Cuanto mayor es el tamaño y la carga de estas especies dopantes más difícil se hace su difusión, favoreciendo su permanencia en el polímero incluso después de reducir el políomero y convertir los híbridos en materiales de inserción catiónica. La actividad electroquímica de los clústers óxidos POM se desarrolla mejor en electrolitos ácidos debido a la protonación simultánea de los clústers durante las reducciones multielectrónicas. Así, su aplicación en celdas reversibles de litio pasa necesariamente por el uso de solventes orgánicos y sales de Li como electrolitos en estas celdas. Este inconveniente no es intrínseco a PMo12 u otros POM pero se deriva de factores experimentales, cosa que deja lugar para la optimización. Por ejemplo, los rápidos y reversibles ciclos de reducción / protonación en los POM hacen de sus híbridos buenos candidatos para otras aplicaciones en el almacenamiento de energía como los supercondensadores. De hecho son posibles muchas otras aplicaciones para este tipo de híbridos derivadas de las propiedades electrónicas, magnéticas, electroquímicas y fotoquímicas de los POM.

$\mathrm{El} \mathrm{Fe}(\mathrm{CN})_{6}$ no presenta ese problema pero no se ancla tan bien en los polímeros, probablemente debido a su menor tamaño. Este anión sólo abandona el híbrido cuando se usan electrolitos orgánicos. En el caso de los electrolitos orgánicos, como los usados normalmente en baterías recargables de litio, el Fe $(\mathrm{CN})_{6}$ permanece en el electrodo.

Desde un punto de vista experimental, el aislamiento de especies moleculares como $\mathrm{POM}$ o $\mathrm{Fe}(\mathrm{CN})_{6}$ dispersas en una matriz sólida, ofrece la posibilidad de hacer química (redox, ácido-base) en un entorno totalmente distinto al de los solventes habituales (agua u orgánicos) o reacciones en estado sólido. Esta aproximación a lo que podríamos llamar "química de matriz sólida" se basaría en la inmovilización real de las moléculas dispersas y la disponibilidad de electrones/ iones, condiciones que se cumplen totalmente en el presente ejemplo de nuestros híbridos.

\section{AGRADECIMIENTOS}

Este trabajo ha sido financiado por CICYT (MAT980807-C02-02). También agradecemos al Ministerio de Educación y Cultura (MEC-AECI) una beca predoctoral concedida a G.T.G. y a CONACYT (México) una beca predoctoral concedida a M.L.C.

\section{BIBLIOGRAFÍA}

1. S.Roth, W.Graupner, "Conductive Polymers: evaluation of industrial applications", Synth.Met., 1993, 55-57, 3623-3631

2. J.S.Miller, "Conducting polymers-Materials of commerce. Part A", Adv. Mater., 5, 587-589 (1993)

3. J.S.Miller, "Conducting polymers-Materials of commerce. Part B", Adv. Mater., 5, 671-676 (1993).

4. A.G.MacDiarmid, "Polyaniline and polypyrrole: where are we headed?", Synth.Met., 84, 27-34 (1997).

5. P.Novák, K.Müller, K.S.V.Santhanam, O.Haas, “Electrochemically Active Polymers for Rechargeable Batteries", Chem.Rev., 97, 207-281 (1997).

6. Pedro Gómez-Romero, Mónica Lira, Nieves Casañ-Pastor. Patent $\mathrm{N}^{\mathrm{o}}$ 9500599, OEPM, Spain (1995).

7. P.Gómez-Romero, M.Lira-Cantú, “Hybrid Organic-Inorganic Electrodes: The Molecular Material Formed Between Polypyrrole and the Phosphomolybdate Anion", Adv.Mater., 9, 144-147 (1997).

8. P. Gómez-Romero, N.Casañ-Pastor, M.Lira-Cantú, "Chemical Polymerization of polyaniline and polypyrrole by phosphomolybdic acid. In situ formation of hybrid organic-inorganic materials", Solid State Ionics, 101-103, 875-880 (1997).

9. M.Lira-Cantú, P.Gómez-Romero, "Electrochemical and chemical syntheses of the hybrid organic-inorganic electroactive Material formed by phosphomolybdate and polyaniline. Application as cation-insertion electrodes", Chem.Mater., 10, 698-704 (1998).

10. M.Lira-Cantú, P.Gómez-Romero, "Cation vs. Anion Insertion in Hybrid Materials Based on Conducting Organic Materials for Energy Storage Applications", Ionics, 3, 194-200 (1997).

11. M.Lira-Cantú, P.Gómez-Romero. "Hybrid Organic-Inorganic Electrodes Formed by Polyoxometalates and Conducting Organic Polymers" in Lithium Polymers Batteries, B.Scrosati Ed, Proceedings of the Electrochem. Soc.Symp., 158-61 (1997)

12. M.Lira-Cantú, P.Gómez-Romero "Hybrid organic-inorganic functional materials: insertion electrodes formed by conducting organic polymers and electroactive inorganic species" in "Recent Research Development in Physical Chemistry", S.G.Pandalai Ed., Transworld Research Network, 379-401 (1997).

M. Lira-Cantú, G. Torres-Gómez and P. Gómez-Romero* “Hybrid Materials based on Conducting Organic Polymers and Electroactive Inorganic Molecules and Oxides. Their Application as Lithium-Insertion Electrodes" Mat.Res.Soc. Symposium Series (Vol. 548). Solid State Ionics V (Editors G.A. Nazri, C. Julien and. A. Rougier) 367-376 (1999).

13. L.L.Miller, B.Zinger, Q.Zhou, "Electrically Controlled Release of $\mathrm{Fe}(\mathrm{CN})_{6}^{-4}$ from Polypyrrole", J.Am.Chem.Soc., 109, 2267-2272 (1987).

14. J.Lei, C.R.Martin, "Infrared investigations of pristine polypyrrole. Is the polymer called polypyrrole really poly(pyrrole-co-hidroxypyrrole)?", Synth.Metals., 48, 331-336 (1992).

15. J.M.Ko, H.W.Rhee, S.M.Park, C.Y.Kim, “Morphology and electrochemical properties of polypyrrole films prepared in aqueous and nonaqueous solvents", J.Electrochem.Soc., 137, 905-909 (1990).

16. P.Novák, "Limitations of polypyrrole synthesis in water and their causes", Electrochim.Acta, 37, 1227-1230 (1992).

17. G.Lian, S.Dong, "Electrochemical behaviour of $\mathrm{Fe}(\mathrm{CN})_{6}^{-3 /-4}$ redox ions in a polypyrrole film", J.Electroanal.Chem., 260, 127-136 (1989).

18. M.Tölgyesi, A.Szücs, C.Visy, M.Novák, “Redox anion doped polypyrrole films: electrochemical behaviour of polypyrrole prepared in $\mathrm{Fe}(\mathrm{CN})_{6}$ solution", Electrochim.Acta, 40, 1127 (1995).

19. B.Zinger, L.L.Miller, "Timed Release of Chemicals from Polypyrrole Films", J.Am.Chem.Soc., 106, 6861-6863 (1984). 